\title{
The correlation between planetary nebula morphology and radio brightness temperatures
}

\author{
J. P. Phillips ${ }^{\star}$ \\ Instituto de Astronomia y Meteorologia, Av. Vallarta No. 2602, Col. Arcos Vallarta, CP 44130 Guadalajara, Jalisco, Mexico \\ Received 18 June 2003 / Accepted 8 August 2003

\begin{abstract}
We have used model central star evolutionary tracks to evaluate the expected variation of $5 \mathrm{GHz}$ brightness temperatures $T_{\mathrm{B}}$ in planetary nebulae. Using these results, and previously derived central star mass functions, we find that there should be a correlation between $T_{\mathrm{B}}$ and the mean central star mass $\left\langle M_{\mathrm{CS}}\right\rangle$, and also between $T_{\mathrm{B}}$ and nebular morphology.

An analysis of the available observational results suggests that such a correlation between $T_{\mathrm{B}}$ and nebular morphology does indeed exist. Bipolar nebulae appear to be concentrated towards high brightness temperatures, whilst circular sources tend to possess lower brightness temperature shells. Elliptical sources are present (and common) at all values of $T_{\mathrm{B}}$. There are upon the progenitor masses of the nebulae. In particular, it is clear that higher mass progenitors are likely to give rise to both bipolar and elliptical outflows; a result which is consistent with analyses of nebular abundances, Zanstra temperatures, and line excitations.
\end{abstract} \\ differences however between the observed and deduced morphological variations, and these enable us to place constraints
}

Key words. planetary nebulae: general - ISM: jets and outflows - stars: evolution

\section{Introduction}

It is now relatively well established that planetary nebulae (PNe) having differing shell morphologies also arise from differing masses of progenitor star. The circular sources are likely to arise from stars somewhat like our Sun, with masses $M_{\mathrm{PG}}$ of order $\sim 1 \rightarrow 1.2 M_{\odot}$. By contrast, the bipolar sources (BPNe) appear to derive from stars which are substantially more massive, and correspond to spectral type $\sim \mathrm{A} 3$ or earlier (see for instance Phillips 2001a; Corradi \& Schwarz 1995).

Finally, it would appear that elliptical sources arise from stars which are intermediate between these two extreme cases, although there is also evidence that they may be ejected from almost any mass of progenitor.

The evidence in support of this scenario has only recently become available. It is now known for instance that the three major morphological classes of PNe (i.e. those having elliptical, circular and bipolar shells) have markedly differing mean heights above the galactic plane (Zuckerman \& Gatley 1988; Manchado et al. 2000; Corradi \& Schwarz 1995; Phillips 2001b). They are also characterized by significant differences in their elemental abundances, line excitations, and Zanstra temperatures (Phillips 2003a-c). All of these factors together imply that shell morphology is linked to progenitor mass $M_{\mathrm{PG}}$ and central star mass $M_{\mathrm{CS}}$ (although see, in addition, the provisos mentioned in Sect. 2).

\footnotetext{
^ e-mail: jpp@udgserv.cencar.udg.mx
}

We shall demonstrate that present models of central star evolution imply a relation between $\left\langle M_{\mathrm{CS}}\right\rangle$ and $T_{\mathrm{B}}$, the $5 \mathrm{GHz}$ brightness temperature, and that there should therefore be a correlation between shell morphology and $T_{\mathrm{B}}$ as well. This latter relationship is confirmed through an analysis of published observations. We shall also find that whilst circular sources tend to derive from lower mass stars, and BPNe from higher mass progenitors, the elliptical PNe appear to arise from all masses of star, and represent a high proportion of sources at all observed brightness temperatures.

\section{The expected relation between brightness temperature and morphology}

We shall aim to investigate the expected variation of nebular morphology with $5 \mathrm{GHz}$ brightness temperature $T_{\mathrm{B}}$. This latter parameter is particularly useful as a measure of shell surface brightness, since it is free from the uncertainties arising from interstellar extinction. There are also many values of $T_{\mathrm{B}}$ available in the published literature (see Sect. 3), permitting a realistic comparison between theoretical and observed trends in morphology.

We make the following assumptions concerning nebular properties:

- The central stars will be assumed to follow H-burning evolutionary tracks. There is now abundant evidence that this is so, as indicated for instance by Schonberner (1989) and Henry \& Shipman (1986). 
- We shall determine H ionizing fluxes $Q(\lambda<912 \AA)$ by using the evolutionary tracks of Schonberner (1979, 1981, 1983) and Blocker \& Schonberner (1990), and by approximating central star continua with Planckian curves. This represents a tolerably good approximation for the present type of analysis (see e.g. Schonberner 1981; Marigo et al. 2001).

- We shall assume the nebular shells to have more-or-less constant velocities of expansion. This is supported by the evidence of shell expansion in Magellanic Cloud planetaries (Stanghellini et al. 1999), and the invariance of observed expansion velocities $V_{\text {EXP }}$ with nebular radius (Phillips 2003d). Although there are systematic differences in velocity between the differing shell morphologies, these appear to be small (Phillips 2002a). Finally, we note that hydrodynamical models of expansion imply both acceleration and deceleration of the I-fronts (see e.g. Schmidt-Voigt \& Koppen 1987; Okorokov et al. 1985; Ferch \& Salpeter 1975; Mellema 1994; Marigo et al. 2001). The mean trend of all of these models taken together is consistent with invariance in $V_{\mathrm{I}}$. Note however that I-front acceleration, similar to that suggested by Marigo et al., makes little qualitative difference to our results or conclusions.

It is expected that PNe envelopes will be optically thick to Lyman continuum radiation during their initial phases of expansion (i.e. that they will be "ionisation bound"). The radio fluxes of the sources are then given by (Pottasch 1984)

$$
\begin{aligned}
F_{v}= & 3.75 \times 10^{-38}\left\{\left[n\left(\mathrm{H}^{+}\right)+n\left(\mathrm{He}^{+}\right)\right] \ln \left[\frac{4.95 \times 10^{-2} T_{\mathrm{e}}^{1.5}}{v}\right]\right. \\
& \left.+4 n\left(\mathrm{He}^{++}\right) \ln \left[\frac{2.47 \times 10^{-2} T_{\mathrm{e}}^{1.5}}{v}\right]\right\} \\
& \times \frac{4}{3} \pi R^{3} \varepsilon T_{\mathrm{e}}^{-0.5} N_{\mathrm{e}} \mathrm{erg} \mathrm{s}^{-1} \mathrm{~Hz}^{-1}
\end{aligned}
$$

where $R$ is the source radius, $v$ is the frequency in $\mathrm{GHz}, \varepsilon$ is the nebular filling factor (the fraction of the nebular volume occupied by gas), $T_{\mathrm{e}}$ is the electron temperature (taken to be $10^{4} \mathrm{~K}$ ), and we take $n\left(\mathrm{He}^{+}\right) / n\left(\mathrm{H}^{+}\right) \cong 0.097$ and $n\left(\mathrm{He}^{++}\right) / n\left(\mathrm{H}^{+}\right) \cong$ 0.013 based upon the tabulated results of Cahn et al. (1992). Given that $R$ corresponds to the Stromgren radii $R_{\text {STROM }}$ of the sources, and that $R_{\text {STROM }}$ depends upon $Q(\lambda<912 \AA)$, it can then be shown that

$$
F(5 \mathrm{GHz}) \cong 1.45 \times 10^{-26} Q(\lambda<912) \mathrm{erg} \mathrm{s}^{-1} \mathrm{~Hz}^{-1}
$$

where we have used case B recombination coefficients.

The envelopes eventually become optically thin as a result of increasing shell sizes and decreasing densities. This leads to shells whose masses $M_{\mathrm{I}}$ are invariant with radius, and given through

$$
M_{\mathrm{I}}=\frac{4}{3} \pi R^{3} n\left(\mathrm{H}^{+}\right) \varepsilon \xi m_{\mathrm{H}}
$$

where $\xi(\cong 1.36)$ is the mean atomic mass per $\mathrm{H}$ atom. If one takes the number of electrons per $\mathrm{H}$ ion to be given by $\gamma=1.12$, then it is readily shown that nebular surface brightness is given through

$S(5 \mathrm{GHz}) \cong \frac{2.27 \times 10^{-24}\left(M_{1} / M_{\mathrm{O}}\right)^{2}}{\varepsilon(R / \mathrm{pc})^{5}} \mathrm{Jy} \mathrm{sr}^{-1}$.
It is still unclear when this latter phase of expansion occurs. The variation of shell masses with radius suggests that many envelopes remain optically thick until quite late in their evolution (e.g. Pottasch 1996). The evidence of Zanstra temperature ratios, on the other hand, appears to suggest that most observed nebulae may be optically thin (Phillips 2003b). Neither these (nor other) analyses are completely decisive. We shall assume, for the present, that shells become optically thin at the point at which central star hydrogen burning desists, and gravo-thermal energy release dominates (Schonberner 1993). The consequences of differing onsets for optically thin expansion are discussed later this section.

Given the assumptions and equations outlined above, it is possible to evaluate the variation in source numbers $N\left(\log \left(T_{\mathrm{B}}(5 \mathrm{GHz})\right)\right.$ as a function of $5 \mathrm{GHz}$ brightness temperature $T_{\mathrm{B}}$, and central star mass $M_{\mathrm{CS}}$. The results are illustrated in Fig. 1 for several cases of $M_{\mathrm{CS}}$. The variations in these profiles result from changes in the ionizing fluxes $Q(\lambda<912 \AA)$, and from variations in the opacity of the nebular shells. The initial narrow peak towards higher values of $T_{\mathrm{B}}$, for instance, occurs where values of $Q(\lambda<912 \AA)$ are at their maximum, whilst the subsequent fall-off to lower temperatures arises from decreased hydrogen burning within the central star. The final increase in $N\left(\log \left(T_{\mathrm{B}}(5 \mathrm{GHz})\right)\right.$ towards the lowest values of brightness temperature (i.e. for values $\log \left(T_{\mathrm{B}}\right)<0$ where $M_{\mathrm{CS}}=0.6 M_{\odot}$ ) is a consequence of optically thin expansion in the nebular shells. This results in a steep initial rise in $N\left(\log \left(T_{\mathrm{B}}\right)\right)$, followed by an exponential increase to lower values of $T_{\mathrm{B}}$.

It is apparent from these trends that the number of sources detected at any particular value of $T_{\mathrm{B}}$ will depend upon the mass of the progenitor star. Where values of $T_{\mathrm{B}}$ are large, then one expects most of the observed sources to be associated with higher central star (and progenitor) masses. This can also be illustrated in a more concrete way.

To do this, we have created a distribution of functions $N\left(\log \left(T_{\mathrm{B}}\right)\right)$ for a synthetic population of masses $M_{\mathrm{CS}}$, based upon trends (and mean profiles) taken from Fig. 1, and profiles for higher and lower central star masses (see Fig. 2). These are weighted according to the central star mass function $N\left(M_{\mathrm{CS}}\right)$ of Phillips (2001a), a trend which is similar to the observationally determined function of Schonberner (as quoted by Weidemann 1989). The lowest central star mass is taken to be $M_{\mathrm{CS}}=$ $0.55 M_{\odot}$, and is represented by the largest amplitude curve (peaking at $\log \left(T_{\mathrm{B}} / K\right)_{\text {PEAK }} \sim 0.4$ ). Subsequent (higher temperature) curves correspond to larger values of $M_{\mathrm{CS}}$, which each curve differing from its neighbours by values $\Delta M_{\mathrm{CS}}$ of between $\sim 0.005 M_{\odot}\left(\right.$ where $M_{\mathrm{CS}} \sim 0.55 M_{\odot}$ and $\left.\log \left(T_{\mathrm{B}} / \mathrm{K}\right)_{\text {PEAK }} \sim 0.4\right)$ and $\sim 0.015 M_{\odot}\left(\right.$ where $M_{\mathrm{CS}} \sim 0.7 M_{\odot}$ and $\log \left(T_{\mathrm{B}} / \mathrm{K}\right)_{\mathrm{PEAK}} \sim$ 3.5). The central star masses are selected so as to lead to an even distribution of curves along the $\log \left(T_{\mathrm{B}}\right)$ axis. Summation of these curves permits us to assess the proportions of differing central star masses for any particular $T_{\mathrm{B}}$, taking into account the increasing effects of opacity as $T_{\mathrm{B}}$ approaches $10^{4} \mathrm{~K}$.

It may be seen that most observed nebulae correspond to lower mass central stars, and that these should represent the dominant component where $\log \left(T_{\mathrm{B}} / \mathrm{K}\right)<1$. On the other hand, it is apparent that higher brightness temperature nebulae tend 


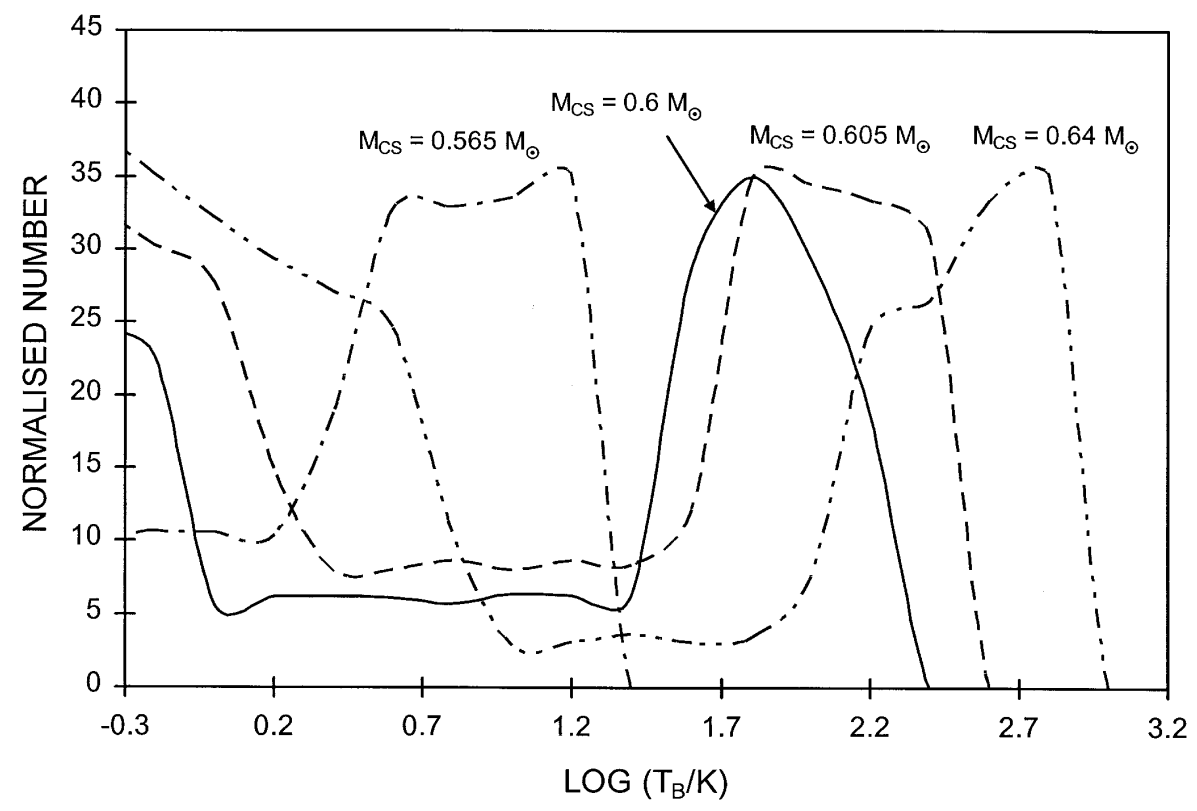

Fig. 1. Comparison between evolutionary trends in $N\left(\log \left(T_{\mathrm{B}}\right)\right)$ for various values of $M_{\mathrm{CS}}$. All of these curves have been arbitrarily normalised. Notice that functions corresponding to higher central star masses are shifted to the right.

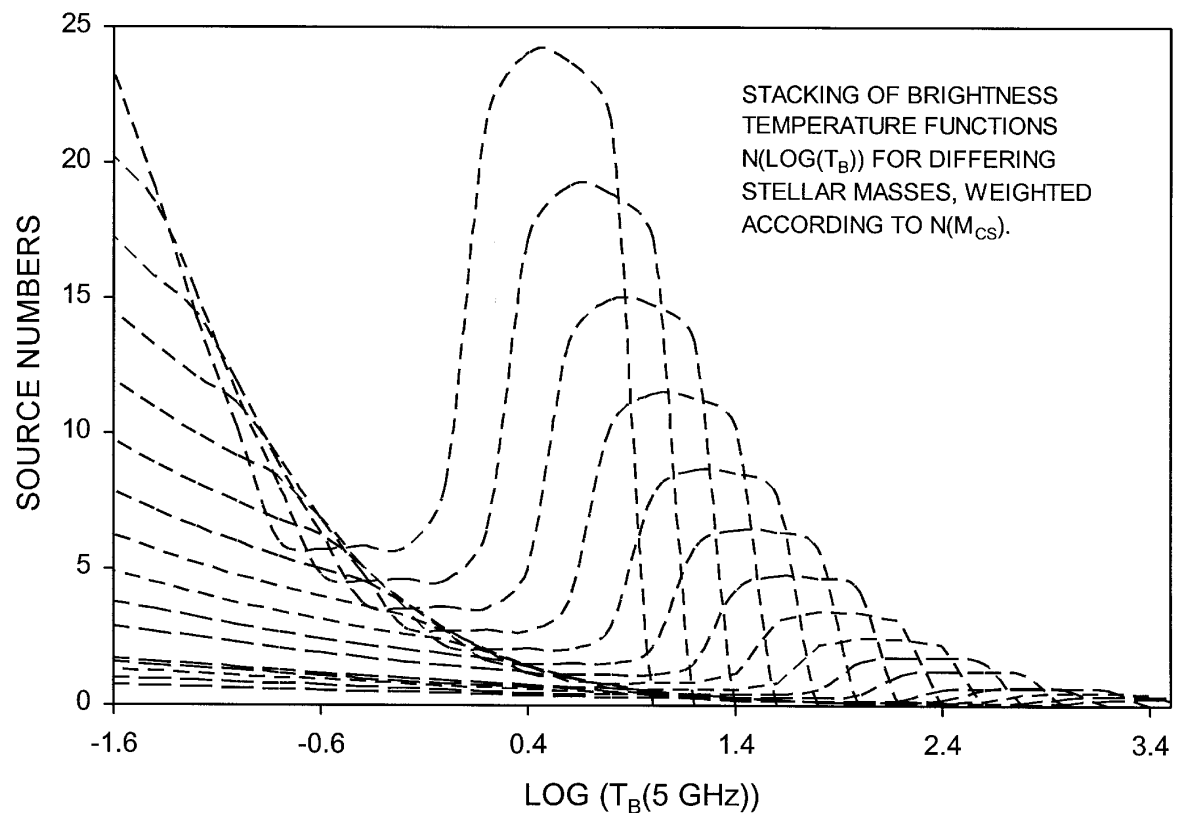

Fig. 2. A stacking of functions $N\left(\log \left(T_{\mathrm{B}}\right)\right)$ for the outflow shells of planetary nebulae. Each curve corresponds to a particular central star mass, and is weighted using the central star mass function of Phillips (2001a). The lowest assumed central star mass is taken to be $M_{\mathrm{CS}}=0.55 M_{\odot}$.

to be associated with higher mass central stars, so that there should be a tendency for $\left\langle M_{\mathrm{CS}}\right\rangle$ to increase with increasing $T_{\mathrm{B}}$.

That this is so is indicated in Fig. 3, where we illustrate the variation of $\left\langle M_{\mathrm{CS}}\right\rangle$ with $\log \left(T_{\mathrm{B}}\right)$. The solid curve corresponds to the case where there is a late onset in optical thinness, as discussed above (case A). The dashed curve corresponds to a case where shells become optically thin when $Q(\lambda<912 \AA)$ reaches its peak (case $B$ ). It may be seen that there is relatively little difference between the respective trends.

We also suspect however that higher mass stars are associated with bipolar nebulae, and lower mass stars with circular outflows (see Sect. 1). Phillips (2001a) has analysed the expected ranges of central star mass for the differing morphologies, and finds that circular sources are likely to occur where $0.55<M_{\mathrm{CS}} / M_{\odot}<0.561$, and bipolar nebulae arise where $M_{\mathrm{CS}}>0.618 M_{\odot}$. He assumes that elliptical outflows are sandwiched between these two extreme cases. There are still continuing doubts about this relationship, however, and it is likely that certain BPNe derive from lower mass progenitors as well (cf. M 2-9, which is identified as a Type III source according to the system of Peimbert (Maciel \& Chiappini 1994)). Similarly, it is likely that central star binarity plays a role in the 


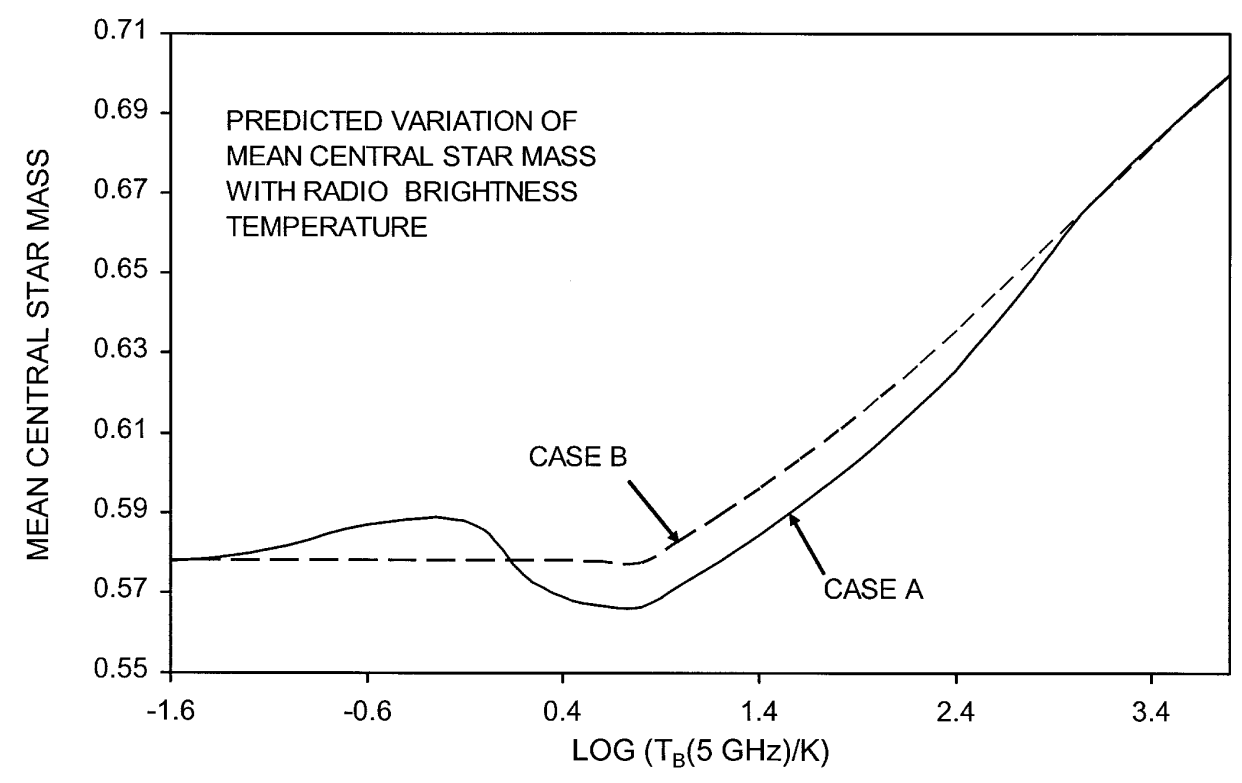

Fig. 3. The variation of mean central star masses $\left\langle M_{\mathrm{CS}}\right\rangle$ with $5 \mathrm{GHz}$ brightness temperature, based upon the analysis described in Sect. 2. We show two cases. It is assumed, in the case of the solid curve, that nebular shells become optically thin during later phases of expansion, when central star hydrogen burning is exhausted (case A). The dashed curve corresponds to the case where shells become optically thin when $\mathrm{H}$ ionizing fluxes $Q(\lambda<912 \AA$ ) are at their maximum (case B). Both trends are closely similar.

formation of bipolar outflows. Taken as a whole, however, it is clear that there are now a broad range of observations support the relationship between $M_{\mathrm{CS}}$ and morphology (see Sect. 1), and it will be assumed that this applies in the proceeding analysis.

Given these mass ranges, and the trends illustrated in Fig. 2, it is then possible to determine the relative populations of the morphologies as a function of $T_{\mathrm{B}}$. This is shown in Fig. 4 for the two cases ( $\mathrm{A}$ and $\mathrm{B}$ ) mentioned above. We have used a relatively low binning resolution for the horizontal axis, so as to permit a later comparison with results determined from observations.

It is clear that bipolar PNe are expected to dominate towards high brightness temperatures, and ellipticals in the range $\log \left(T_{\mathrm{B}} / \mathrm{K}\right) \sim 1 \rightarrow 2.2$. It is also likely that circular and elliptical sources will have a similar frequency of occurrence where brightness temperatures are low. There is little qualitative difference between the trends for cases A and B.

We shall determine, in the following sections, whether these model predictions are confirmed through observations.

\section{The data base}

Recent investigations of nebular morphologies now enable us to identify circular, bipolar, and elliptical shells in a large number of planetary nebulae. Manchado et al. (1996) have evaluated the morphological characteristics of $243 \mathrm{PNe}$, for instance, whilst similar investigations have been undertaken by Hromov \& Kohoutek (1968), Stanghellini et al. (1993), Soker (1997) and Zuckerman \& Aller (1986). Listings of bipolar nebulae may additionally be found in Corradi \& Schwarz (1995) and Schwarz et al. (1992). Some care requires to be undertaken in concatenating these various data sets, however. Thus, it is clear that whilst the type I sources of Hromov \& Kohoutek (1968) correlate well with the circular sources listed in other surveys, this is not the case for their category II and III elliptical outflows. Many of these latter sources have subsequently been found to correspond to bipolar outflows, and we have therefore discarded all of these nebulae in assessing mean trends in nebular morphology. On the other hand, we note that socalled multi-polar (or quadripolar) sources such as NGC 2440 and M2-46 appear generically similar to the bipolar outflows, and are often categorised as such. We have therefore binned all such nebulae with the BPNe.

Most of these morphological investigations are in close agreement, and appear to imply similar classifications in $\sim 85 \%$ of cases. One exception to this is the analysis of Soker (1997), however, who finds a relatively small proportion of circular PNe. We have not included his results in the present analysis.

Finally, we have employed the listing of $5 \mathrm{GHz}$ brightness temperatures provided by Phillips (2002b). These are similar to the values listed by Zhang (1995), and are determined using radio interferometric dimensions $\theta_{\mathrm{RAD}}$ listed by Isaacman (1984), Phillips \& Mampaso (1988), and Zijlstra et al. (1989). Where such dimensions are not available, then we have used optical diameters $\theta_{\mathrm{VIS}}$ quoted by Cahn et al. (1992) and Cahn $\&$ Kaler (1971). A comparison between these differing sets of dimensions suggests that $\left\langle\theta_{\mathrm{RAD}}\right\rangle \cong 1.04\left\langle\theta_{\mathrm{VIS}}\right\rangle$; that is, that both sets of diameters are similar in the mean.

It is also clear, for the larger part of the sources considered here, that $\theta_{\mathrm{RAD}}$ and $\theta_{\mathrm{VIS}}$ correspond to the brightest parts of the nebular shells. Where one is dealing solely with circular or elliptical PNe, then it is probably fair to assume that these angular dimensions correspond to the overall source sizes - excepting, that is, for envelopes having secondary halos. This is not however the case for bipolar nebulae. For these cases, the faint 

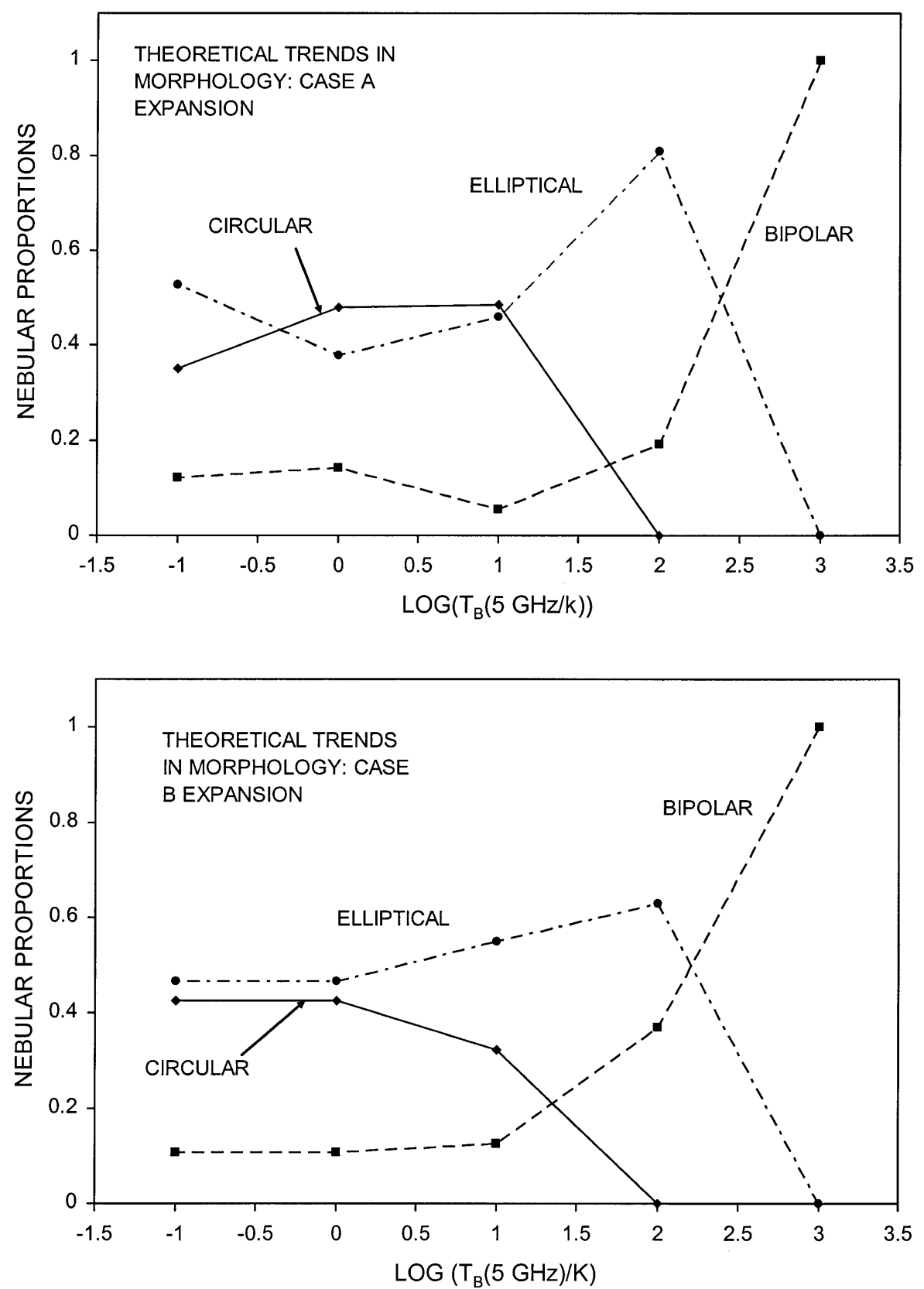

Fig. 4. The variations in the proportions of differing nebular morphologies predicted using the current analysis. The upper panel refers to case A expansion, whilst the lower panel corresponds to case B (see the text for details, and also the caption to Fig. 3). It will be noted that whilst there are differences between the curves, their qualitative appearance is similar.

bipolar extensions often surmount what are much more compact and brighter nebular cores, and our values for brightness temperature apply to these latter structures alone.

Cross comparison between the references cited above enables us to identify $N=171$ sources having $5 \mathrm{GHz}$ brightness temperatures, and circular $(N($ CIRC $)=69)$, elliptical $(N($ ELLIPT $)=63)$ and bipolar $(N(\mathrm{BPNe})=39)$ morphologies. These numbers are biased however as a result of the limited delectability of sources at $5 \mathrm{GHz}$, and do not reflect intrinsic trends in source numbers. The number of elliptical sources appears to be similar to those having circular morphologies, for instance, when in fact $N$ (ELLIPT) is much greater than $N(\mathrm{CIRC})$ for PNe taken as a whole (Phillips 2001a). To take account for this observational bias, we therefore define a function

$\Phi(\mathrm{CIRC})=$

$$
\frac{N_{\mathrm{TOT}}(\mathrm{CIRC})}{N_{\mathrm{TOT}}(\mathrm{CIRC})+N_{\mathrm{TOT}}(\mathrm{ELLIPT})+N_{\mathrm{TOT}}(\mathrm{BPNe})}
$$

corresponding to the intrinsic fraction of PNe having circular morphologies. $N_{\mathrm{TOT}}(\mathrm{CIRC}), N_{\mathrm{TOT}}(\mathrm{ELLIPT})$ and $N_{\mathrm{TOT}}(\mathrm{BPNe})$ correspond to the total observed numbers of the differing morphologies. Similar relations apply for bipolar and elliptical PNe. The proportion $P(\mathrm{CIRC})$ of circular PNe within 


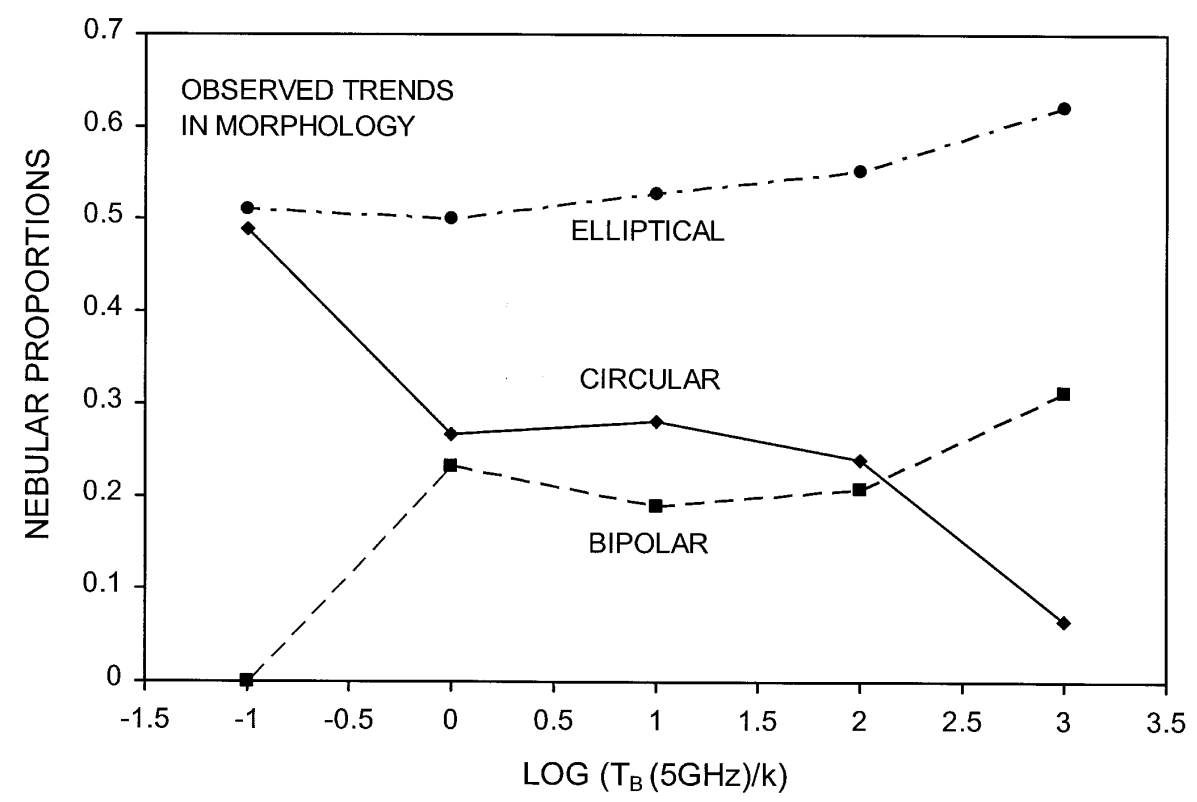

Fig. 5. The observed proportions of differing nebular morphologies as a function of $5 \mathrm{GHz}$ brightness temperature.

any particular brightness temperature bin $\Delta \log \left(T_{\mathrm{B}}\right)$ is then given by

$$
\begin{gathered}
P(\mathrm{CIRC})=\Phi(\mathrm{CIRC}) N_{\mathrm{BIN}}(\mathrm{CIRC})\left(\Phi(\mathrm{CIRC}) N_{\mathrm{BIN}}(\mathrm{CIRC})\right. \\
\left.+\Phi(\mathrm{ELLIPT}) N_{\mathrm{BIN}}(\mathrm{ELLIPT})+\Phi(\mathrm{BPNe}) N_{\mathrm{BIN}}(\mathrm{BPNe})\right)^{-1}
\end{gathered}
$$

where $N_{\mathrm{BIN}}(\mathrm{CIRC}), N_{\mathrm{BIN}}(\mathrm{ELLIPT})$, and $N_{\mathrm{BIN}}(\mathrm{BPNe})$ are the numbers of circular, elliptical and bipolar sources detected within the bin. $P($ CIRC) therefore represents the proportion of circular nebulae detected within a particular brightness temperature bin, weighted to reflect the intrinsic incidence of these sources. We have determined values of $\Phi$ based upon the results of Phillips (2001a). A graph of the variation of $P$ with $T_{\mathrm{B}}$ is illustrated in Fig. 5.

One final point is worth noting. Whilst the bipolar, circular and elliptical outflows constitute the majority of PNe, they do not represent all such sources. Roughly $17 \%$ of nebulae fall outside of these morphological sub-groups. Since these latter sources form a pretty mixed group of structures, with little underlying consistency in their morphologies, and since we have little idea of what their progenitor masses might be, we have chosen to excise these nebulae from the present analysis.

\section{Comparison of theoretical and observational trends}

It is clear from Fig. 5 that there is, as expected, a trend for the proportions of differing morphologies to vary with brightness temperature $T_{\mathrm{B}}$. The profile for the bipolar nebulae peaks towards high values of $T_{\mathrm{B}}$, and falls-off sharply below $\log \left(T_{\mathrm{B}} / K\right) \sim 0$. The circular sources follow a reverse trend, peaking at low values of $T_{\mathrm{B}}$ and decreasing towards higher values. Finally, elliptical outflows appear to be common at all values of $T_{\mathrm{B}}$, although they are proportionally rather more prevalent towards higher brightness temperatures.
How good is the comparison between these trends and the results of the analysis in Sect. 2? A superficial comparison between Figs. 4 and 5 suggests that the differences are in fact considerable. We do not expect there to be any elliptical sources where $\log \left(T_{\mathrm{B}}\right) \sim 3$, for instance, whereas the proportion of observed sources appears to peak at this value. Similarly, the incidence of BPNe is lower than expected where $T_{\mathrm{B}}$ is low, whilst circular sources extend to much higher brightness temperatures than is theoretically expected.

What do these differences imply? The first important point to note is that the observed trends are not entirely free of error. Probably the most important of these arises from socalled "morphological cross-over", whereby bipolar and elliptical sources may be confused with those having circular morphologies. Bipolar nebulae may appear circular when viewed pole-on, whilst elliptical sources may also appear circular when viewed along their axes of symmetry. The proportion of circular sources is therefore likely to appear larger than is in reality the case.

Such tendencies are likely to explain the observed presence of circular sources towards higher values of $T_{\mathrm{B}}$.

A somewhat similar occurrence takes place where values of $T_{\mathrm{B}}$ are low. In this case, not only may BPNe appear circular when viewed pole-on, but the lobes may be too faint to detect at all (given that their visual surface brightnesses will also tend to be reduced). This is exacerbated in the case of these latter sources because of their low mean galactic latitudes, and the tendency for fainter nebular features to be "obscured" by IS extinction. The brighter nuclei of these sources may therefore be confused with circular or elliptical PNe.

This has two main consequences. The first is that the proportion of BPNe is likely to appear much smaller than is the case where $T_{\mathrm{B}}$ is low, and this could explain the zero incidence of such sources where $\log \left(T_{\mathrm{B}} / K\right) \cong-1$. The second is that the proportion of circular and elliptical sources will again increase. 
It is clear from the above, therefore, that care requires to be taken in the interpretation of the results. Nevertheless, it is apparent that various trends exist which are difficult to interpret in these terms alone. Thus, the relatively modest incidence of BPNe towards higher values of $T_{\mathrm{B}}$ is in direct conflict with theoretical expectations, and cannot be attributed to morphological cross-over. This suggests that elliptical sources may, in reality, be generated by higher mass progenitors. If this is the case (and it is difficult to imagine many other explanations for this trend), then one would require $\sim 60 \%$ of such stars give rise to elliptical nebulae, and $\sim 30 \%$ to BPNe. This effect, and the role of morphological cross-over, are probably sufficient to account for all of the disparities between the theoretical and observed distributions.

It should finally be noted that elliptical sources are likely to arise from a broad range of masses; larger, almost certainly, than has been supposed by Phillips (2001a) (see the comments in Sect. 1, and later this section). It therefore follows that the lower limit mass $M_{\mathrm{CS}}$ for bipolar sources may be less than supposed above (i.e. less than $\cong 0.618 M_{\odot}$ ), and the mass range for circular nebulae may be somewhat greater. This would lead to a broadening in the ranges of $T_{\mathrm{B}}$ over which the various morphologies can be detected. Similarly, we have taken the transition time $t_{\mathrm{TR}}$, the period between when the central star leaves the AGB tip and nebulae become appreciably ionized, to be of relatively short duration. Where this is not the case, then it may result in a shift in our curves to lower values of $T_{\mathrm{B}}$. Unless $t_{\mathrm{TR}}$ varies strongly with progenitor mass, however, then the deduced trends in morphology are likely to remain unchanged.

There are therefore several main conclusions which can be drawn from the present analysis:

- One expects, on the basis of the analysis in Sect. 2, that sources having high brightness temperatures will tend to be associated with larger progenitor and central star masses.

- Given that there is a relation between $\left\langle M_{\mathrm{CS}}\right\rangle$ and $T_{\mathrm{B}}$, one then predicts a variation in the proportions of differing morphologies as a function of $T_{\mathrm{B}}$, as illustrated in Fig. 4 . Although the precise form of this relationship is open to some tweaking, and depends upon uncertain details of nebular expansion, there seems little doubt that the derived results are likely to be qualitatively correct.

- An analysis of observed trends in morphology also implies a correlation between shell morphology and $5 \mathrm{GHz}$ brightness temperature, as illustrated in Fig. 5.

- Comparison between the observed and predicted trends (i.e. those illustrated in Figs. 4 and 5) implies that a good proportion of higher mass progenitors give rise to elliptical outflows. It is also apparent that elliptical outflows are common at all values of brightness temperature, implying that the parent stars must have a broad range of masses.

This latter result is of particular interest, since it confirms the conclusions arrived at by other independent analyses. Phillips (2003a) finds that abundances in elliptical outflows trespass upon the ranges characterising circular and bipolar PNe, implying that elliptical nebulae are likely to arise from all masses of star. Similarly, it has been found that the range in Zanstra temperatures $T_{\mathrm{Z}}(\mathrm{He}$ II) for these sources is reasonably broad, implying progenitor masses $\sim 1 \rightarrow 2.7 M_{\odot}$ (Phillips 2003b).

Finally, it has been noted that elliptical nebulae have the widest range of emission line ratios of any class of nebula, which is again suggestive of a broad range of progenitor masses (Phillips 2003c).

There is therefore increasing evidence that elliptical sources arise from a large range of progenitor masses, a result which appears also to be supported by our present results.

\section{Conclusions}

We have used the expected evolutionary variations in central star ionising fluxes to demonstrate the likely presence of strong biases in nebular brightness temperatures. High brightness temperature sources are more likely to be associated with higher mass central star (and progenitor) masses, and vice versa for sources having low brightness temperatures. Given that there is also a correlation between progenitor mass and nebular morphology, one then anticipates that there should be a relation between brightness temperature and morphology as well.

That this is so is borne out by our present results. We show that bipolar sources tend to be located towards higher values of $T_{\mathrm{B}}$, whilst circular sources tend to be concentrated towards lower brightness temperatures. Elliptical outflows appear to constitute a similar (and large) proportion of $\mathrm{PNe}$ at all values of $T_{\mathrm{B}}$.

Comparison of these trends with theoretical expectations leads to several conclusions. It is apparent, in the first place, that some of the observed trends are likely to be affected by morphological cross-over, and this may explain the zero incidence of BPNe towards the lowest values of $T_{\mathrm{B}}$, and an apparent extension of circular nebulae towards higher values of $T_{\mathrm{B}}$.

Such tendencies cannot however explain why most high $T_{\mathrm{B}}$ sources appear to be elliptical, and the proportion of BPNe is relatively modest. We therefore conclude that a good proportion of higher mass progenitors are likely to give rise to both bipolar and elliptical envelopes.

This result is in excellent accord previous studies of elemental abundance, line excitation, and He II Zanstra temperatures.

\section{References}

Acker, A., Ochsenbein, F., Stenholm, B., et al. 1992, Strasbourg-ESO Catalogue of Planetary nebulae (Garching: ESO)

Blocker, T., \& Schonberner, D. 1990, A\&A, 240, L11

Cahn, J. H., \& Kaler, J. B. 1971, ApJS, 22, 319

Cahn, J. H., Kaler, J. B., \& Stanghellini, L. 1992, A\&AS, 94, 399

Corradi, R. L. M., \& Schwarz, H. E. 1995, A\&A, 293, 871

Ferch, R. L., \& Salpeter, E. E. 1975, ApJ, 202, 195

Henry, R. B. C., \& Shipman, H. L. 1986, ApJ, 311, 774

Hromov, G. S., \& Kohoutek, L. 1968, BAC, 19, 1

Isaacman, R. 1984, MNRAS, 208, 399

Maciel, W. J., \& Chiappini, C. 1994, Ap\&SS, 219, 231

Manchado, A., Guerrero, M. A., Stanghellini, L., \& Serra-Ricart, M. 1996, The IAC Morphological Catalog of Northern Planetary nebulae (Spain: IAC)

Marigo, P., Girari, L., Groenwegen, M. A. T., \& Weiss, A. 2001, A\&A, 378,958 
Mellema, G. 1994, A\&A, 290, 915

Okorokov, V. A., Shustov, B. M., Tutukov, A. V., \& Yorke, H. W. 1985, A\&A, 142, 441

Phillips, J. P. 2001a, MNRAS, 32, 1041

Phillips, J. P. 2001b, PASP, 113, 839

Phillips, J. P. 2002a, A\&A, 393, 1027

Phillips, J. P. 2002b, ApJS, 139, 199

Phillips, J. P. 2003a, MNRAS, in press

Phillips, J. P. 2003b, MNRAS, in press

Phillips, J. P. 2003c, A\&A, submitted

Phillips, J. P. 2003d, Astron. Nach., in press

Phillips, J. P., \& Mampaso, A. 1988, A\&A, 190, 237

Pottasch, S. R. 1984, Planetary Nebulae (Dordrecht: Reidel)

Pottasch, S. R. 1996, A\&A, 307, 561

Schmidt-Voigt, M., \& Köppen, J. 1987, A\&A, 174, 211

Schonberner, D. 1979, A\&A, 79, 108

Schönberner, D. 1981, A\&A, 103, 119
Schonberner, D. 1983, ApJ, 272, 708

Schonberner, D. 1989, in Planetary Nebulae, ed. S. Torres-Peimbert, IAU Proc. Symp., 131 (Dordercht: Kluwer), 464

Schonberner, D. 1993, in Planetary Nebulae, ed. R. Weinberger, \& A. Acker, IAU Proc. Symp., 155, 415 (Dordercht: Kluwer)

Schwarz, H. E., Corradi, R. L. M., \& Melnick, J. 1992, A\&AS, 96, 23 Soker, N. 1997, ApJS, 112, 487

Stanghellini, L., Blades, J. C., Osmer, S. J., Barlow, M. J., \& Liu, X.-W. 1999, ApJ, 510, 687

Stanghellini, L., Corradi, R. L. M., \& Schwarz, H. E. 1993, A\&A, 279,521

Weidemann, V. 1989, A\&A, 213, 155

Zhang, C. Y. 1995, ApJS, 98, 659

Zijlstra, A. A., Pottasch, S. R., \& Bignell, C. 1989, A\&AS, 79, 329

Zuckerman, B., \& Aller, L. H. 1986, ApJ, 301, 772

Zuckerman, B., \& Gatley, I. 1988, ApJ, 324, 501 